\title{
THE SIRENS OF TITAN: POSMODERNISMO Y LA CONDICIÓN HUMANA
}

Diego Sheinbaum Lerner*

RESUMEN: En el artículo se delinean los alcances artísticos de The sirens of Titan, de Kurt Vonnegut, ${ }^{1}$ y su capacidad para explorar los dilemas de la cultura posmoderna y de la condición humana tal como fue formulada por Hannah Arendt. Se propone que la versatilidad en el manejo de tramas, personajes, mundos posibles y del narrador le permite a Vonnegut plasmar los rasgos distintivos de la cultura estadounidense de la posguerra, al mismo tiempo que desnuda sus fundamentos filosóficos por medio del uso virtuoso de la parodia.

yode

\section{THE SiRENS OF Titan: PostModernism AND THE HuMAN CONDITION}

ABSTRACT: The article delineates the artistic scope of Kurt Vonnegut's The Sirens of Titan, and his ability to explore the dilemmas of postmodern culture and the human condition as formulated by Hannah Arendt. It suggests that the versatility in the handling of plots, characters, possible worlds and the narrator allows Vonnegut to capture the distinctive features of post-war U.S. culture, while he strips its philosophical foundations through the virtuous use of parody.

PALABRAS CLAVE: Vonnegut, posmodernismo, Arendt, parodia, era espacial.

KEY WORDS: Vonnegut, postmodernism, Arendt, parody, space age.

RECEPCIÓN: 4 de agosto de 2016. APROBACIÓN: 5 de junio de 2017.

* Investigador del Centro de Poética, UNAM.

${ }^{1}$ The sirens of Titan, 1959, Nueva York, Dial Press Trade Paperbacks. 
CITAM Derechos Reservados.

La reproducción total o parcial de este artículo se podrá hacer si el ITAM otorga la autorización previamente por escrito. 


\section{THE SIRENS OF TITAN: POSMODERNISMO Y LA CONDICIÓN HUMANA}

No es una coincidencia que la carrera literaria de Kurt Vonnegut despegue al unísono del posmodernismo y la era espacial. Si bien su inicio profesional se remonta diez años atrás, a la serie de relatos publicados en revistas comerciales, solo con la aparición en 1959 de su segunda novela, The sirens of Titan, alcanza un virtuosismo que lo coloca entre los escritores de primera fila. Sin embargo, a diferencia de autores como John Barth y Thomas Pynchon, resulta asombroso que la escritura de Vonnegut se vuelva penetrante, fresca y vanguardista, no a través de la lectura crítica de la tradición literaria, sino como respuesta a sus experiencias personales y a las condiciones de vida en Estados Unidos. Su posmodernismo, para decirlo de otra manera, es más difícil de explicar como respuesta al agotamiento de artificios modernistas (modelo formalista) o como parte de una identificación de grupo que intenta abrirse un espacio cultural (modelo sociológico) y es más comprensible como respuesta a los cambios sociales y a las transformaciones en el conocimiento (modelo cognitivo). ${ }^{1}$

En este sentido, quizá vale la pena resaltar tres experiencias vitales que marcan su literatura. Primera, durante su infancia Vonnegut atestiguó el duro golpe que la Gran Depresión le dio a su familia. No es extraño, por lo mismo, encontrar que sus novelas abordan el problema del trabajo,

${ }^{1}$ Douwe Fokkema, "The semiotics of literary postmodernism", en International postmodernism: Theory and literary practice, 1997, Ámsterdam, J. Benjamins, p. 18. 
el dinero y la especulación. Segunda, durante su juventud estudió ingeniería mecánica y ciencias, y después vivió en carne propia los usos que se les dieron en la Segunda Guerra Mundial. Como prisionero de guerra, sobrevivió al bombardeo aliado que destruyó Dresde. Esta experiencia yace explícitamente en el corazón de su quinta novela, SlaughterhouseFive, e implícitamente en toda su obra. Por último, en su edad madura trabajó unos años como publicista de General Electric, donde fue parte del nuevo corporativismo que se forjó en las décadas de 1940 y 1950. Así, la cultura empresarial y el aparato administrativo encontraron también un lugar en su narrativa. ${ }^{2}$

Estas experiencias pusieron las bases para que la obra de Vonnegut, y en particular The sirens of Titan, explore la naciente cultura estadounidense de la posguerra, analizada muchas veces con el término de posmodernismo. En esta novela, la parodia de ciencia ficción traza las mutaciones del espacio y del tiempo, del sujeto y del cambio, descritas por Frederic Jameson como parte de la lógica del capitalismo tardío. Sus dos protagonistas sufren desterritorializaciones: Malachi pierde la memoria en Marte, naufraga y enloquece en Mercurio, es confundido con el mesías de la religión del Dios completamente indiferente en la Tierra y es desterrado a Titán. Por su parte, Rumfoord vive condenado a materializarse y desmaterializare cada 59 días en la Tierra.

Vonnegut utiliza la parodia de ciencia ficción para ir más allá de la división entre cultura de masas y alta cultura que caracterizó al modernismo y cuya indistinción marcará la estética posmoderna. ${ }^{3}$ Es una parodia, como escribió Linda Hutcheon, de doble filo. ${ }^{4}$ Ataca la ciencia ficción, desnudando la ridiculez de sus disparatadas tramas, y simultáneamente, despliega los elementos del género para activar motivos muy serios: sondear el agotamiento de la trascendencia, todavía importante para la poética modernista; arremeter contra el elitismo en que se

${ }^{2}$ Jerome Klinkowitz, The Vonnegut effect, 2011, Columbia, University of South Carolina Press, pp. 1-19.

${ }^{3}$ Andreas Huyssen, After the great divide. Modernism, mass culture, postmodernism, 1986, Indianápolis, Indiana University Press.

${ }^{4}$ Linda Hutcheon, A poetics of postmodernism: History, theory, fiction, 1988, Nueva York, Routledge, p. 126. 
empantana el proyecto moderno (el de los hombres, blancos, ricos y occidentales); transparentar las endebles ilusiones del humanismo liberal, y mostrar la bancarrota de los relatos que apuntan hacia un fin último de la humanidad. ${ }^{5}$

Estos rasgos emergen a lo largo de su novela, pero no son los más notorios. Su obra está mucho más cerca de las conceptualizaciones del posmodernismo literario que han hecho Douwe Fokkema y Brian McHale. Por una parte, Sirens tiene el sello de un existencialismo desplazado, desprovisto de la angustia y que da lugar al "anything goes", ${ }^{6}$ $\mathrm{y}$, por la otra, explora estas cuestiones no desde el encierro, sino a través de la expansión del universo, por lo que no se queda en el solipsismo modernista que tantea dilemas epistemológicos de una conciencia encarnada, sino que su poética, marcada por lo ontológico, se abre a la lógica de mundos posibles y a las nuevas formas de ser que suponen. ${ }^{7}$

En este sentido, me parece que la manera en que aborda la provisionalidad de nuestro mundo - la manera en que se disuelve y se crea constantemente frente a nuestros ojos- está muy cerca de reflexiones filosóficas como las de Hannah Arendt. El escritor estadounidense se pone en esta ruta, impulsado por su versatilidad narrativa y paródica. Con múltiples tramas, personajes y mundos, su literatura se expande, mientras que su genio cómico la reduce a las características esenciales. Gracias a esta destreza narrativa, atraviesa las diferentes capas del posmodernismo hasta alcanzar una altitud que abre una perspectiva casi filosófica sobre las nuevas condiciones tecnológicas, las cuales apuntan a una transformación profunda y elemental en la relación entre la humanidad y el planeta.

Esto se percibe, sobre todo, en sus personajes, que son sometidos a las maravillosas y terribles consecuencias que supone la introducción de fuerzas universales, en el sentido literal del término, en la Tierra. ${ }^{8}$

${ }^{5}$ Hans Bertens, "Introduction”, International postmodernism, op. cit., pp. 3-18; Jean François Lyotard, La condición postmoderna: Informe sobre el saber, 1990, México, Rei.

${ }^{6}$ Fokkema, op. cit., pp. 21-22.

${ }^{7}$ Brian McHale, The Cambridge introduction to postmodernism, 2015, Nueva York, Cambridge University Press.

${ }^{8}$ Hannah Arendt, The human condition, 1958, Chicago, Chicago University Press. 
Estas fuerzas liberan las condiciones que han marcado la vida del hombre durante milenos y rompen las cadenas que lo unían al trabajo, a la enfermedad, al dolor y a una vida breve, pero también le dan la oportunidad de borrar la vida del planeta. Se trata del amanecer de una nueva época; sin embargo, a diferencia de otros amaneceres, los rayos no necesariamente provienen del espacio, como lo demostraron las radiaciones de Hiroshima y Nagasaki.

La parodia es fundamental para lograr todo esto. Porque si en Sirens la multiplicación de artificios lleva a Vonnegut a estas alturas, la parodia le permite desprenderse de ellos y empujar los límites de la narración hasta descubrirse en una región donde la comedia y la broma bordean el dolor y el absurdo. Ahí el narrador encuentra palabras que rozan las de una filosofía primera, en la que lo metafísico, lo ontológico, lo ético, lo religioso y lo político no están separados. Es una región donde solo se formulan preguntas elementales: ¿Qué es el mundo? ¿Qué implica ser humano? ¿Es posible la justicia? ¿Existe la libertad?

\section{Tramas desorbitadas y parodias terrenales}

En cada una de sus novelas, Vonnegut nos recuerda que el negocio de la ficción, como diría Truman Capote, consiste en la mezcla de lo extraordinario y lo improbable. Estas son las palancas para alzar el caparazón de la banalidad y encontrar el sentido. ${ }^{9}$ Para eso, Vonnegut utiliza la ciencia ficción como unos siglos antes Cervantes utilizó los libros de caballerías. En su segunda novela, estas palancas se convierten en plataformas de despegue que mandan la banalidad y el sentido por los cielos. Los ponen a girar en torno a la Tierra.

Vonnegut inyecta el sentido de lo extraordinario en Sirens desde el inicio de la trama. Winston Niles Rumfoord, uno de los personajes centrales, se materializa cada 59 días en la Tierra como parte de su descomposición en el tiempo y el espacio, provocada por haber dirigido

${ }^{9}$ Stephen Koch, Modern library writer's workshop, 2003, Nueva York, The Modern Library, p. 80 . 
su nave hacia un infundibulum cronosinclástico. Esto le permite leer la mente de otros y ver el futuro, por lo que hace profecías que parecen improbables. Este material, de por sí alucinante, es llevado al extremo a través de artilugios melodramáticos: vuelcos del destino, hombres que descubren que su identidad es otra, malos que se vuelven buenos, cartas con revelaciones, mensajes cifrados y ejecuciones públicas.

Resulta llamativa la maestría para construir artilugios y multiplicarlos. El elenco completo es revolucionado por mecanismos melodramáticos: de ser un vendedor de artículos de cocina, Noel Constant se convierte en un especulador multimillonario; Boaz pasa de ser huérfano en la Tierra a ser general en Marte, después trunca su sadismo y se convierte en amoroso cuidador de harmoniums. Malachi, el más frívolo, libertino y parásito de los multimillonarios, termina en Titán como un hombre autosuficiente que limpia altares. Por último, Rumfoord, quien parece mover los hilos de estos destinos y encarnar al gran manipulador, termina por darse cuenta de que es el gran manipulado.

Junto a los vuelcos dramáticos se multiplican profecías, encuentros excepcionales, mensajes y cartas. Rumfoord predice que su esposa, Beatriz, y Malachi, el hombre más rico de Estados Unidos, van a viajar a Marte, se van a aparear, van a regresar a la Tierra y de ahí se irán a vivir a una luna de Saturno. La religión del Dios completamente indiferente predice la llegada del vagabundo del espacio, las palabras exactas que dirá y la talla del traje que le quedará a la perfección. A esto hay que agregar la manera y las condiciones en que los personajes se encuentran. Ramson Fern llega a decirle a Noel Constant, quien no lo conoce, que Noel lo va a contratar, le va a pagar dos mil dólares y van a construir un emporio.

El sentimentalismo que normalmente acompaña a los artilugios melodramáticos es reemplazado por un acento paródico que los activa $\mathrm{y}$, al mismo tiempo, los vacía, dejando una estela. Así, en vez de la materialización de un simple hombre, tenemos la de un hombre y su perro (el mastín del espacio); en vez de la profecía de un matrimonio, la de un simple apareamiento; las palabras anunciadas por la profecía y proferidas por el vagabundo del espacio no están cargadas de emoción y sentido, 
sino son las más simples y vacías. El traje que le queda a la perfección es ridículamente amarrillo y tiene un signo de interrogación.

Si Northrop Frye especulaba que el movimiento de cualquier trama imita los ritos de pasaje entre el invierno y la primavera, la muerte y la resurrección, el aislamiento y la comunión, en la novela de Vonnegut estos ciclos adquieren un ritmo frenético, como si los propios destinos humanos se hubieran acelerado con la era espacial. La disparidad entre elementos continuos está creada, no a través de un pequeño salto, sino de uno cuántico; no de un ligero desfase, sino de uno que tiene una escala planetaria. Un hombre que Malachi nunca ha visto le dice que han estado juntos en una luna de Saturno; nuestro protagonista no termina en Colono, sino exiliado a millones de años luz. El efecto hiperbólico, desarmado por la parodia, se vuelve terrenal, mundano, incluso demoniaco. Salo ha sido escogido para llevar un mensaje del distante Trafalmadore hasta la otra orilla de la galaxia; sin embargo, cuando desobedece las órdenes y lee el mensaje, descubre que solo dice "Greetings".

La veta paródica encuentra su cauce tradicional en la crítica de la trascendencia y, en especial, de la religión. A veces se trata de guiños; por ejemplo, el método de Noel Constant para amasar una fortuna consiste en utilizar la Biblia para seleccionar las empresas de las cuales va a comprar acciones. Otras veces se trata de la reescritura de pasajes bíblicos. Una de las situaciones más logradas es cuando el reverendo Denton condena el orgullo vano de los hombres y el hambre de poder que los ha llevado a construir naves para asaltar los cielos. La referencia natural es a la Torre de Babel. El genio narrativo de Vonnegut consiste no solo en encontrar la extraña analogía entre la Tierra y una nave espacial, sino en montar una serie de paralelismos que penetran hasta los más mínimos detalles. ${ }^{10}$

10 "You want to fly through the space? God has already given the most wonderful space ship in all creation! Yes! Speed? You want speed? The space ship God has given you goes sixty-six thousand miles an hour - and will keep on running at that speed for all eternity, if Gods wills it. You want a space ship that will carry men in comfort? You've got it! It won't carry just a rich man and his dog or just five or just ten men. No! God is no pike! He's given you a space ship that would carry billions of men, women, and children! Yes! And they don't have to stay strapped in chairs or wear fishbowls over their head. No! Not on God's space ship. The people on God's space 


\section{Un elenco existencialista toma por asalto el cielo}

El reparto de Sirens parece una instancia genial de la mutación que Fokkema apreció en el posmodernismo como un existencialismo desplazado: sin la angustia y el callejón del suicidio. Los personajes de Vonnegut se enfrentan a problemas existenciales como la libertad y la ausencia de sentido. La mayoría está buscando algo que complete su vida. Esperan convertirse en mensajeros de un significado que le dé sentido a su existencia. Entre ellos sobresale Malachi, consciente de que su nombre quiere decir "el mensajero fiel" hasta el extremo de que ha mandado a hacer un escudo de armas que dice "El mensajero espera". Si bien su comportamiento no parece adecuarse a este papel, en las depresiones que siguen invariablemente a sus abusos de alcohol, narcóticos y mujeres anhela solo una cosa: un mensaje lo suficientemente digno e importante para que merezca que él lo lleve entre dos puntos. ${ }^{11}$

Malachi solo es la punta de lanza de un conjunto de mensajeros sin mensajes, personajes que buscan una pieza que complete el sentido, herederos galácticos de Kafka. Cuando Malachi queda en la bancarrota, su administrador, Ransom Fern, cumple su último deber, darle la carta que el padre de Malachi le pidió que le entregara en caso de que se le acabara la suerte. Antes de irse, Fern le pide un último favor: "If the letter seems to cast the vaguest light on what life might be about, I would appreciate you telephoning me at home". ${ }^{12}$ Incluso los trafalmadorianos mandan mensajes a la máquina, Salo, para que no se desespere mientras llega la refacción de su nave. Desde Titán, Salo lee estos mensajes en la superficie de la Tierra. Se trata de grandes construcciones como Stonehenge, la Muralla China, la casa dorada de Nerón, el Kremlin y el edificio de Naciones Unidas. Estos mensajes ponen de relieve, junto a la búsqueda de sentido, la manipulación y la falta de libertad de los humanos. Vonnegut convierte el fin último de la historia de la civilización — representada por estas construcciones paradigmáticas - en la

ship can go swimming, and walk in the sunshine and play baseball and go ice skating and go for family rides in the family automobile on Sunday." The sirens of Titan, p. 28.

${ }^{11}$ Ibid., p. 12.

${ }^{12}$ Ibid., p. 83. 
entrega de una pequeña refacción para que una máquina extraterrestre pueda arreglar su nave y llevar un mensaje al otro lado de la galaxia, cuyo contenido es solo un saludo.

Winston Niles Rumfoord es el único que parece escapar a esta situación. Winston y Malachi están orquestados como dobles contrarios. Los dos son multimillonarios estadounidenses: Winston, un aristócrata de la costa este con un sentido del honor y lo ético notable; Malachi, un especulador financiero de Hollywood, vulgar y mujeriego. Ambos tienen todo lo que uno puede pedir: dinero, belleza y talento. En este sentido, nos llevan a esa dimensión, más profunda y elemental, de la nueva relación del hombre con la Tierra. Ambos pertenecen a las primeras generaciones en la historia de la humanidad que viven como dioses, sin las cadenas de la necesidad. Malachi representa a la mayoría de los nuevos afortunados, entregados a una fiesta permanente. No es para menos: la emancipación parece total.

Sin embargo, el problema, como lo ha dicho Michel Serres, es que en nuestro mundo la necesidad, en vez de desaparecer, cambió de bando. Si la libertad y necesidad se entendían como contrarios, de pronto la necesidad aparece en el mismo campo de la libertad. El nuevo poder nos vuelve responsables de todo. Ni los dioses ni la naturaleza explican ahora las hambrunas, la falta de agua o las explosiones demográficas. Todo esto queda en nuestras manos. ${ }^{13}$ Es el precio de la libertad, pero ¿quién está dispuesto a levantarse de la fiesta para preocuparse de los nuevos problemas?

Wiston Niles Rumfoord parece el tipo de hombre forjado durante generaciones para enfrentar estos nuevos retos. Winston concibe un plan para hacerle frente a la dispareja repartición de la suerte que convierte a unos pocos hombres en triunfadores (millonarios, guapos y talentosos) y a la inmensa mayoría en perdedores. Por supuesto, no se trata de un problema menor. Nunca en la historia de la humanidad han habido tan pocos ganadores y tantos perdedores hundidos en la necesidad. El talento de Vonnegut radica en juntar a estos dos hombres opuestos en

${ }^{13}$ Michel Serres, Conversations on science, culture, and time, 1995, Ann Arbor, University of Michigan Press. 
condiciones excepcionales, diálogos que ocurren en el breve lapso entre materializaciones y que le permiten ir al corazón de la cuestión.

Así, a diferencia del coro existencialista, Rumfoord busca que sus acciones le den sentido a su vida. Su meta no parece muy diferente a la que inspirará a John Rawls a escribir Una teoría de la justicia y a formular el velo de la ignorancia como una forma de equiparar la carrera de la vida antes de que esta inicie. Pero si el objetivo de Winston es liberal y de sentido común, no así los medios que adoptará. Quizá es la sensatez de Rawls la que cae en oídos sordos y los humanos solo nos decidimos a cambiar nuestro rumbo político cuando nos enfrentamos a lo insensato. Rumfoord utiliza la guerra de Marte contra la Tierra y el remordimiento — que la acompaña como una breve resaca — para fundar la religión del Dios completamente indiferente. El rito principal de este nuevo credo consiste en que los afortunados usen obstáculos que los incapaciten:

There were women who had received by dint of dumb luck the terrific advantage of beauty. They had annihilated that unfair advantage with frumpish clothes, bad posture, chewing gum, and a ghoulish use of cosmetics [...] One old man, whose only advantage was excellent eyesight, had spoiled that eyesight by wearing his wife's spectacles [...] A dark young man, whose lithe, predaceous sex appeal could not be spoiled by bad clothe and bad manners, had handicapped himself with a wife who was nauseated by sex. ${ }^{14}$

La ironía, que tiñe a la religión del Dios completamente indiferente, desarma las buenas intenciones de Rumfoord y muestra lo descabellado de su proyecto. De esta manera, Vonnegut vacía el sueño de justicia. La libertad de acción se rebela como un espejismo. Los personajes principales son marionetas al servicio de Rumfoord. Por más que se rebelan contra su destino, acaban sucumbiendo. Rumfoord no es la excepción. En las últimas páginas descubre que fue manipulado por los trafalmadorianos. El cuestionamiento de la acción humana y de la

${ }^{14}$ The sirens of Titan, pp. 227-228. 
libertad alcanza su punto más alto en una nueva variación de la historia de la humanidad, que es narrada por una máquina en el registro de un cuento infantil:

Once upon a time on Trafalmadore there were creatures who weren't anything like machines. They weren't dependable. They weren't efficient. They weren't predictable. They weren't durable. And these poor creatures were obsessed by the idea that everything that existed had to have a purpose, and that some purposes were higher than others. These creatures spent most of their time trying to find out what their purpose was. And every time they found out what seem to be a purpose of themselves, the purpose seemed so low that the creatures were filled with disgust and shame. ${ }^{15}$

El planteamiento es genial, pero Vonnegut lo llevará hasta sus últimas consecuencias. Antes de seguirlo, vale la pena señalar que a lo largo de la novela el autor yuxtapone diversos registros: sermones, adivinanzas, himnos militares, cartas, recetas, leyendas, cuentos para niños, enciclopedias, canciones mnemotécnicas y libros académicos. En muchas ocasiones, como en el párrafo anterior, Vonnegut mezcla registros con tonos dispares que provocan grandes contrastes. Aquí el cuento de niños se transforma en una parábola filosófica. El cuestionamiento ataca el centro de la libertad y de la ética: la idea de que hay propósitos y que unos pueden ser más altos que otros. Al mismo tiempo, Vonnegut delinea la naturaleza humana comparándola con la eficiencia, confianza y predictibilidad de las máquinas. La relación casi ontológica de los seres humanos con los propósitos y la relación práctica con las máquinas le permiten cambiar de motores y elevar su especulación a una altura que produce vértigo:

And, rather than served such low purpose, the creatures would make a machine to serve it. This left the creatures free to serve higher purposes. But whenever they found a higher purpose, the purpose wasn't high enough. So machines were made to serve higher purpose, too.

${ }^{15}$ Ibid., p. 279. 
And the machines did everything so expertly that they were finally given the job of finding out what the highest purpose of the creatures could be. The machines reported in all honesty that the creatures couldn't really be said to have any purposes at all. The creatures thereupon began slaying each other because they hated purposeless things above else. And the machines finished up the job in less time than it takes to say Trafalmadore. ${ }^{16}$

Con el final, Vonnegut regresa al registro de cuento para niños, no sin antes hacer explotar la parábola filosófica, de tal manera que a la pesadilla extrema y sanguinaria le sigue el dulce sueño infantil. Así concluye su visión apocalíptica de la evolución tecnológica como si fuera hora de apagar la luz e irse a dormir.

\section{Mundos posibles en la era espacial}

Es sintomático que, para entrar a la residencia de los Rumfoord, Malachi atraviese una pequeña puerta de Alicia en el país de las maravillas, ya que su vagabundeo por el espacio será, entre otras cosas, un viaje psicodélico por mundos paralelos, con leyes y seres particulares. También en este sentido la obra de Vonnegut es paradigmática del posmodernismo. McHale ha vinculado las referencias a Alicia con la exploración de mundos posibles que caracterizan a esta literatura. ${ }^{17}$

Pronto, en el viaje de Malachi, los elementos extraños se tornan alucinatorios. Junto con él nos descubrimos en Marte, en medio de una ejecución pública. Unk (el nuevo nombre de Malachi) ha estado en el hospital, donde le borraron la memoria y ahora es dirigido por una antena que le colocaron en el cerebro, para que ahogue, con sus propias manos, al traidor, ante la mirada de los batallones. El condenado a muerte parece conocerlo y, antes de que lo mate, alcanza a decirle que la carta está escondida en una de las últimas barracas. Unk logra escabullirse y encuentra la carta. Descubre que él y su amigo, Stony Stevenson,

${ }^{16}$ Ibid., pp. 279-280.

${ }^{17} \mathrm{McHale}$, op. cit., pp. 50-55. 
han apuntado lo que conocen sobre ellos y el mundo de Marte para recuperar la memoria, en caso de que sean sometidos a los tratamientos.

Con este artificio, Vonnegut nos lleva de nuevo hacia una investigación ontológica del mundo y el hombre. La carta consiste en un listado de las cosas que saben, sobre las cuales tienen certidumbre y que comienza con un reconocimiento cartesiano de la ignorancia y de la importancia de las preguntas: "They aren't much — but here are the things that I know for sure [...] The questions are important. I have thought harder about them than about the answers I already have. That is the first thing that I know for sure: (1) If the questions don't make sense, neither will the answers. [...] The second item that I know: (2) I am a thing called alive". ${ }^{18}$

El listado reconstruye el mundo desde lo más elemental, por medio de una notoria habilidad narrativa para fusionar la caricatura, lo filosófico y lo religioso. Esta reconstrucción primaria contrasta con el tono, mucho más experimental e iconoclasta, que evoca el narrador al describir Mercurio; de tal manera que si Marte es una versión del totalitarismo, con ejércitos formados por hombres sin memoria, gobernados por antenas que les infligen dolor en caso de desobediencia, Mercurio es una versión psicodélica existencialista.

La exploración de estos mundos involucra un cambio de escala que marcará la literatura de Vonnegut. Se trata de una expansión del horizonte que la propia novela asocia con el descubrimiento de América. Esta alteración de la perspectiva supone una nueva relación con la Tierra. Por lo menos, es lo que argumenta Hannah Arendt al ver en la era espacial una culminación de la era moderna. Para ella, en el umbral de esta época se produjeron tres grandes acontecimientos que determinan su carácter: el descubrimiento de América y la subsiguiente exploración de la Tierra completa; la Reforma que, a través de la expropiación de posesiones eclesiásticas y monásticas, comenzó el proceso de expropiación individual y acumulación de riqueza social; y la invención del telescopio, que supuso el desarrollo de una nueva ciencia que considera la naturaleza de la Tierra desde el punto de vista del universo.

${ }^{18}$ The sirens of Titan, p. 124. 
Paradójicamente, la transformación que tuvo el mayor impacto fue la que menos pareció tenerlo a los ojos de sus contemporáneos: la adición de un nuevo implemento al ya largo arsenal de herramientas, sin utilidad salvo para mirar las estrellas. Estos fueron los primeros pasos hacia el descubrimiento del universo. El instrumento le permitió al hombre ganar distancia sobre la Tierra y, también, estableció las condiciones para su alienación del entorno terrenal inmediato. La era espacial consumó este proceso. Los experimentos tecnológicos y científicos hicieron posible tratar a la naturaleza desde la perspectiva del universo. Nos permitieron viajar al punto que el telescopio había descubierto y actuar sobre la Tierra como si dispusiéramos de ella desde afuera, incluso a riesgo de poner en peligro la vida natural y de exponer el planeta a fuerzas universales, cósmicas, ajenas. ${ }^{19}$

Así, al mismo tiempo que llegamos a otros planetas, nuestro mundo común se disolvió en una transformación constante. Sometimos a los objetos, que se interponen entre nosotros, a una mutación frenética, de tal manera que la estabilidad que era la característica de nuestro mundo se disolvió. La estabilidad y la dureza del mundo se volvieron provisionales; la distancia entre los mundos reales y los mundos posibles se desdibujó. Los primeros efectos de esta disolución se sienten en Sirens, pero en Slaughterhouse-Five se vuelven una característica fundamental de la obra de Vonnegut.

\section{El telescopio del narrador}

Antes de dar pie a la estrambótica historia de Sirens, la primera operación que Vonnegut realiza consiste en poner de relieve la situación del narrador y el destinatario, de tal manera que vuelve evidente que es tan importante en los géneros narrativos como la relación entre el actor y el público en el teatro. El narrador es el mediador, "el espíritu de la historia", una de las grandes invenciones de la imaginación. Tradicionalmente, por medio del narrador, el autor se disfrazaba de una gran

\footnotetext{
${ }^{19}$ Arendt, op. cit., pp. 248-251.
} 
autoridad que trascendía al simple humano. Era capaz de hablar de las más distintas personas y de sus más sentidos secretos. Podía moverse sin restricciones en el tiempo y en el espacio. ${ }^{20}$ Vonnegut retoma la libertad de estos narradores; sin embargo, su singularidad radica en la distancia que encuentra para ver los asuntos humanos, en el cambio de escalas que, como un telescopio, nos arroja de lo personal a lo cósmico, de lo cotidiano a las últimas cuestiones, de las palabras coloquiales a las palabras del mito y la filosofía. Si el credo modernista, siguiendo a Henry James, había recalcado la importancia de las perspectivas subjetivas - si a través de la famosa fórmula de "The house of fiction has many windows", se había regodeado en la relatividad y yuxtaposición de sensibilidades e inteligencias - , la literatura posmoderna de Vonnegut muestra que, junto a las ventanas, hay observatorios astronómicos que cambian por completo los dilemas.

En Sirens, el lugar del narrador se pone de relieve en la historia marco que envuelve a las peripecias de los personajes. En el inicio, el narrador explica el tipo de historia que relatará. Estos primeros párrafos establecen la distancia entre el tiempo de la narración y el tiempo de lo narrado. Lo hacen con un tono filosófico, con una premisa cautivadora y completamente ficcional:

EVERYONE NOW KNOWS how to find the meaning of life within himself. But mankind wasn't always so lucky. Less than century ago men and women did not have easy access to the puzzle boxes within them.

They could not name even one of the fifty-three portals to the soul.

Mankind, ignorant of the truths that lie within every human being, looked outward - pushed ever outward. What mankind hoped to learn in its outward push was who was actually in charge of all creation, and what all creation was all about. ${ }^{21}$

Vonnegut se establece en los terrenos de la filosofía al escoger como personaje principal a la humanidad. El tono elemental está en conso-

${ }^{20}$ Roy Pascal, Kafka's narrators. A study of his stories and sketches, 1982, Cambridge, Cambridge University Press, pp. 1-2.

${ }^{21}$ The sirens of Titan, p. 1. 
nancia con la extensión de los párrafos, que muchas veces no pasan de una línea. Por obra de la concisión, el gran aliento y el sentido irónico encapsulan el tiempo, el conocimiento y la distancia desde la que hablará el narrador. Palabras que pertenecen al vocabulario de la religión y de la filosofía (el sentido de la vida, el alma y la mirada interior) ganan énfasis a través de la repetición, pero también son desarmadas al combinarlas con un vocabulario que proviene de la ingeniería. Si los primeros cuatro párrafos insisten en la búsqueda interior, los siguientes nos relatan el movimiento contrario:

Mankind flung its advance agents outward, ever outward. Eventually it flung them out into space, into the colorless, tasteless, weightless sea of outwardness without end.

It flung them like stones.

These unhappy agents found what had already been found in abundance on Earth - a nightmare of meaninglessness without end. The bounties of space, of infinite outwardness, were three: empty heroics, low comedy, and pointless death. ${ }^{22}$

Es curioso que Vonnegut perciba el mismo anhelo de huida de la Tierra que formuló Hannah Arendt. Es un anhelo desesperado, que se denota en la elección del verbo "flung”, en su repetición y culminación en el símil con las piedras. La caracterización de este mundo exterior como un lugar vacío se vuelve más enfática por los tres adjetivos que terminan en "less". La búsqueda épica culmina en la ironía macabra de encontrar lo que ya tenían en abundancia. Por lo mismo, estos adjetivos se han convertido en la propiedad característica de este universo: el sinsentido. El vínculo se estrecha por la evocación del infinito ("without end"). Los tesoros, aquí mencionados, son los que explota Vonnegut a lo largo de su obra: heroísmo vacío, comedia baja y muerte sin sentido. La comedia baja está relacionada con los motivos del dinero, riquezas, sexo, envidias y enojos. El narrador de Vonnegut se mueve entre ellos y la distancia telescópica propia de un lenguaje filosófico. Esta se siente en el paso de la historia marco a la historia concreta de los protagonistas.

${ }^{22} I b i d .$, p. 1. 
La transición se vuelve distintiva por la manera en que el mundo va apareciendo poco a poco, a través de trazos que hacen sentir el acto de creación de manera más fuerte, más dramática, porque las letras no construyen la escena de inmediato, sino que la arman poco a poco, al mismo tiempo que llaman la atención sobre el vacío que la rodea: There was a crowd. ${ }^{23}$

Con esta oración párrafo comienza propiamente la historia. Hay que advertir, por última vez, la elegancia para hacer el cambio de escala. Se trata de una especie de zoom que nos lleva de la epopeya general de la humanidad a esta situación en la que una multitud básica, desnuda, sin adjetivos, surge de la nada. El bosquejo está tan cerca de lo bíblico como de la broma y de la premisa lógica al estilo de Wittgenstein. La agilidad del narrador será una de las principales conquistas de Vonnegut en The sirens of Titan. Su relación íntima con un estilo elemental y repetitivo se nota en el contexto, que impulsa la anécdota más allá de los límites de la narración.

No es la única transgresión de los límites de la narración. Se traspasan de nuevo en el juego con los epígrafes al comienzo de cada capítulo. Vonnegut los enarbola para desdibujar la frontera entre el interior

74 y el exterior del texto mediante frases de los propios personajes, como si fueran autores famosos. Son las primeras maniobras de una invasión de lo paratextual que se extenderá en futuras novelas. Se trata menos, en cualquier caso, del deliberado boicot contra la tradición literaria y más de la expansión natural de un genio fabulador que no cabe en los confines de la página. Estos artilugios se suman a la versatilidad del narrador, el manejo de tramas, personajes y mundos, para llevar a Vonnegut por los avatares de la sociedad estadounidense de la segunda mitad del siglo Xx y, sobre todo, por el cambio de la condición humana. Todo esto, gracias a que utiliza la parodia como un traje de astronauta, para traspasar registros, géneros y respirar en las regiones extremas de nuestra atmósfera emocional.

${ }^{23}$ Ibid., p. 2. 